\title{
Gold Nanorod-Mediated Photothermal Modulation for Localized Ablation of Cancer Cells
}

\author{
Yoochan Hong, ${ }^{1}$ Eugene Lee, ${ }^{2}$ Jihye Choi, ${ }^{3}$ Seung Jae Oh, ${ }^{2,4,5}$ Seungjoo Haam,, 4 \\ Yong-Min Huh, ${ }^{2,4,6}$ Dae Sung Yoon, ${ }^{1}$ Jin-Suck Suh, ${ }^{2,4,6}$ and Jaemoon Yang, ${ }^{2,5}$ \\ ${ }^{1}$ Department of Biomedical Engineering, Yonsei University, 234 Maeji, Heungup, Gangwondo, Wonju 220-710, Republic of Korea \\ ${ }^{2}$ Department of Radiology, College of Medicine, Yonsei University, Sinchon, Seodaemun, Seoul 120-752, Republic of Korea \\ ${ }^{3}$ Department of Chemical and Biomolecular Engineering, Yonsei University, Sinchon, Seodaemun, Seoul 120-749, Republic of Korea \\ ${ }^{4}$ YUHS-KRIBB Medical Convergence Research Institute, Sinchon, Seodaemun, Seoul 120-752, Republic of Korea \\ ${ }^{5}$ Severance Integrative Research Institute for Cerebral \& Cardiovascular Diseases, Yonsei University Health System, \\ Seoul 120-752, Republic of Korea \\ ${ }^{6}$ Severance Biomedical Science Institute (SBSI), Shinchon, Seodaemun, Seoul 120-752, Republic of Korea
}

Correspondence should be addressed to Jin-Suck Suh, jss@yuhs.ac and Jaemoon Yang, 177hum@yuhs.ac

Received 15 August 2012; Accepted 20 September 2012

Academic Editor: Haiya Li

Copyright () 2012 Yoochan Hong et al. This is an open access article distributed under the Creative Commons Attribution License, which permits unrestricted use, distribution, and reproduction in any medium, provided the original work is properly cited.

We estimated the photothermal transduction efficiency of gold nanorod (GNR) solutions for different GNR concentrations and irradiation laser power. In particular, we verified that the degree of cell death area could be modulated by GNR concentration and irradiation laser power. The efficacy of GNR-produced photothermal ablation of cancer cells was evaluated by irradiating GNRs in the presence of MDA-MB-231 breast cancer cells with a near-infrared (NIR) laser at different laser power densities and irradiation times. GNR-induced photothermal ablation was applied successfully to cancer cells at various NIR laser power densities and irradiation times and was characterized with live-dead cell staining. Through these techniques, we established the system for not only verification of induced photothermal effect using NIR laser and thermocouple, but also identification of uptake efficiency for GNRs and cell viability using dark field and fluorescence imaging, respectively.

\section{Introduction}

Photothermal cancer therapy using nanostructures has been an attractive technique for the localized ablation of target cancer cells in a minimally invasive manner [1]. Nearinfrared (NIR) light is particularly useful for photothermal therapies because biological tissues are relatively transparent to NIR light [2]. To maximize the photothermal effects, it is important to manufacture well-tailored nanostructures that can absorb NIR light well to transform into heat for localized target cell ablation [3-5]. Recently, there have been many photothermal studies using various nanostructures such as carbon nanotubes [6], gold nanoparticles (GNPs) [7], and gold nanorods (GNRs) [8]. Among them, GNRs are particularly attractive because their longitudinal surface plasmon band is tunable by adjusting the aspect ratio of the rod (length/width) [9]. The longitudinal surface plasmon band for GNRs is located in the NIR region. Photon excitation of the surface plasmon band of GNRs by NIR light oscillates the free electron cloud. The kinetic energy of the oscillating electrons is then conveyed to the lattice of GNRs through electron-phonon coupling. Accordingly, the lattice also oscillates, and the GNRs reach thermal equilibrium with their environment through heat dissipation [9]. Although substantial efforts have been devoted to photothermal therapy, most previous research has focused on target cell viability after photothermal agent internalization and NIR light exposure and has neglected characterization of the influence of specific conditions, for example, light power density, concentration of agents, and light exposure time, on the generation of heat and the subsequent temperature changes.

In this study, we modulated photothermal effects of GNRs solutions subjected to NIR laser irradiation under 
various conditions (i.e., changing power density of NIR laser and GNR concentration). Results were compared to those using GNP solutions as a control photothermal agent. The temperature elevation rates were estimated for each concentration of GNR and GNP solution. The rate of temperature elevation and the photothermal transduction efficiency (the ratio of the energy converted to heat to the energy delivered from by the NIR laser) were measured for each sample. Finally, GNR solutions with high photothermal transduction efficiency were used to ablate MDA-MB-231 breast cancer cells at various NIR laser irradiation powers and durations. Using a series of these processes, we developed the versatile system that can measure the temperature elevation rate of solutions, detect the uptake of photothermal agents, and diagnose the cell viability.

\section{Experimental Methods}

2.1. Preparation of Gold Nanorods (GNRs). Monodispersed GNRs were synthesized by a seed-mediated growth method according to a previously published protocol, with some modification [10]. In brief, for the preparation of gold seed solution, $0.25 \mathrm{~mL}$ of $\mathrm{HAuCl}_{4} \cdot 3 \mathrm{H}_{2} \mathrm{O}(100 \mathrm{mM})$ and $7.5 \mathrm{~mL}$ of hexadecyltrimethylammonium bromide (CTAB) solution $(95 \mathrm{mM})$ were mixed, and $0.06 \mathrm{~mL}$ of ice-cold sodium borohydride solution $(100 \mathrm{mM})$ was added to the mixture under vigorous stirring. The solution mixture was allowed to react for 2 minutes and was stored at room temperature for 3 hours. A growth solution containing $80 \mu \mathrm{L}$ of silver nitrate solution $(100 \mathrm{mM})$ was prepared by addition of $0.5 \mathrm{~mL}$ of $\mathrm{HAuCl}_{4} \cdot 3 \mathrm{H}_{2} \mathrm{O}(100 \mathrm{mM})$ and $9.5 \mathrm{~mL}$ of CTAB solution $(95 \mathrm{mM})$ under vigorous stirring. After the addition of $55 \mu \mathrm{L}$ of ascorbic acid solution $(100 \mathrm{mM})$, the color of the solution changed from yellow to colorless. $12 \mu \mathrm{L}$ of gold seed solution was introduced into the growth solution and stirred for 10 seconds. The product solution was maintained for 24 hours at room temperature without disturbing, and its color changed from colorless to reddish-brown as time went on. The GNR solutions were centrifuged twice at $15,000 \mathrm{rpm}$ for 30 minutes to remove excess CTAB molecules and were then redispersed in $5 \mathrm{~mL}$ of deionized water.

2.2. Preparation of Gold Nanoparticles (GNPs). Monodispersed GNPs were also synthesized using a previously published protocol as a control photothermal agent for comparison to GNRs [11]. In brief, GNPs were prepared by the reduction of $1.0 \mathrm{wt} \%$ tetrachloroaurate(III) trihydrate $(2 \mathrm{~mL})$ in the presence of $80 \mathrm{wt} \%$ tetrakis(hydroxymethyl) phosphonium chloride $(12 \mu \mathrm{L})$ and $\mathrm{NaOH}(0.5 \mathrm{~mL}$ of $1 \mathrm{M})$ as reducing agents for 10 minutes at room temperature.

2.3. Preparation of PEGylated GNRs (PGNRs). Monodispersed PGNRs were prepared using a previously published protocol [12]. To prepare PGNRs, GNRs were coated with heterobifunctionalized PEG (CM-PEG-SH) as a stabilizer. $50 \mathrm{mg}$ of CM-PEG-SH was added to $5 \mathrm{~mL}$ of GNR solution and stirred for 48 hours at room temperature. The mixture was centrifuged at $15,000 \mathrm{rpm}$ for 30 minutes to remove unbound CM-PEG-SH molecules and resuspended in $5 \mathrm{~mL}$ of PBS.

2.4. Characterization of Photothermal Agents. The absorbance of GNRs and GNPs was measured using UV-Vis spectrometer (UV1800, Shimadzu). The chemical structure of the PGNRs was analyzed using a proton-nuclear magnetic resonance $\left({ }^{1} \mathrm{H}-\mathrm{NMR}\right)$ spectrometer (JUM-ECP300, JEOL Ltd.) with dideuterium oxide as solvent. The quantities of $\mathrm{Au}$ in GNRs and GNPs were determined using inductively coupled plasma atomic emission spectrometry (ICP-AES, Thermo Electron corporation).

2.5. Photothermal Effect Measurement. To prepare samples for photothermal effect studies, $320 \mu \mathrm{L}$ GNR solutions $(0.0295,0.0394,0.0492$, and $0.0591 \mathrm{mg} / \mathrm{mL})$ and GNP solutions $(0.0125,0.025,0.05$, and $0.1 \mathrm{mg} / \mathrm{mL})$ were prepared in glass vials. The samples were kept at room temperature for at least 30 minutes prior to irradiation to establish a steady-state temperature. The sample vials were enclosed within styrofoam vial racks and were irradiated with an 808 $\mathrm{nm}$ diode laser (JOLD-30-FC-12, JENOPTIK) with a $1 \mathrm{~mm}$ beam diameter for one minute at power densities of 0.25 , $0.5,1.0$, or $2.0 \mathrm{~W} / \mathrm{cm}^{2}$, respectively. Temperature elevations of GNR and GNP solutions irradiated with a NIR laser were measured using thermocouple (True RMS Multimeter 287, Fluke).

2.6. Cellular Uptake. The uptake efficiency of PGNRs by MDA-MB-231 cells was assessed from dark field images using a high numerical aperture dark field condenser (UDCW, Olympus), which delivers a very narrow beam of white light from a tungsten halogen lamp to the surface of the sample.Immersion oil (nd: 1.516, Olympus) was used to narrow the gap between the condenser and the glass slide and to balance the refractive index. The dark field images were captured using an Olympus CCD camera.

2.7. Photothermal Ablation of Cancer Cells. To test the photothermal ablation potential of the GNRs, MDA-MB231 breast cancer cells $\left(5 \times 10^{4}\right.$ cells/well $)$ were incubated using a previously published protocol [9]. The distributions of the live and dead cells were observed using an fluorescence imaging system based on commercial microscopic system (BX51, Olympus) after cellular staining with an acetomethoxy derivative of calcein (calcein AM: live cells) and ethidium bromide (EthBr: dead cells).

\section{Results and Discussion}

The absorption peaks of GNRs at 520 and $780 \mathrm{~nm}$ (UV1800, Shimadzu) due to the oscillation of electrons along the transverse and longitudinal axes of the nanorod, respectively (Figure 1), were in good agreement with previous studies [13]. In contrast to GNRs, GNPs, which maintain a more uniform geometry, only had a peak at $520 \mathrm{~nm}$. Collectively, these results suggest that GNRs have the potential for more efficient photothermal conversion than GNPs in the NIR region. 


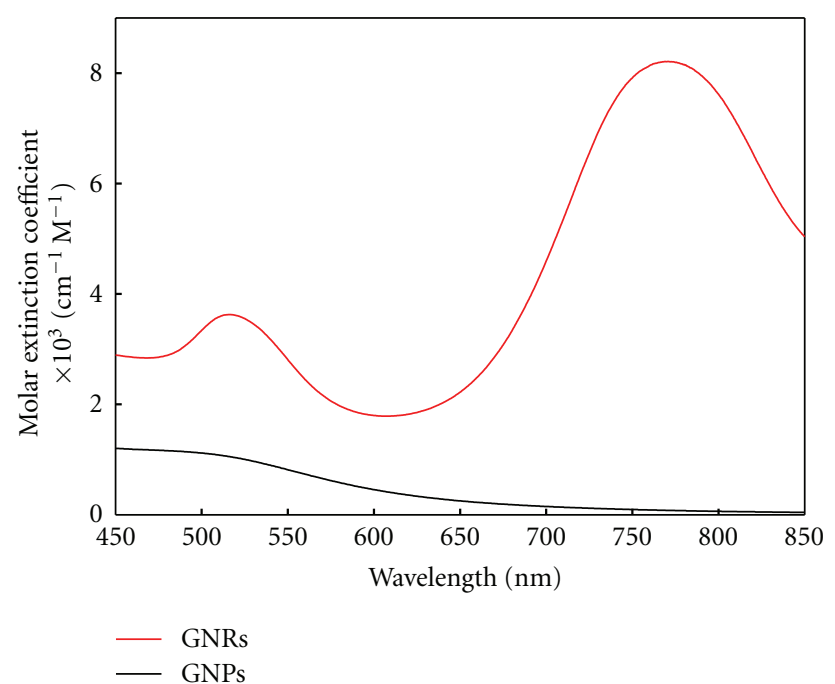

FIGURE 1: Molar extinction coefficient spectra of GNR and GNP solutions.

To investigate the photothermal effects of the samples, first, we designed optical system that optimized for irradiation of NIR laser. A schematic illustration of the temperature measurement system is shown in Figure 2(a). Styrofoam vial racks insulated the samples from ambient temperature variations to improve the accuracy of the measurement of temperature elevations. Temperature elevation rates were estimated from temperature differences during the initial 10 seconds of each experiment. The sample temperatures increased almost linearly with power density. Greater temperature increases were observed with GNR solutions at higher GNR concentrations and higher NIR laser power density, whereas the temperature of GNP solutions increased almost the same degree regardless of the GNP concentration. Linear fits of the temperature elevation rate of each GNR and GNP solution as functions of irradiated power density confirmed these observations (Figure 2(b)).

The photothermal transduction efficiency for each sample was also estimated using previously published calculation method [14]. First, the energy received by each solution used $Q=c m \Delta T$ (where $Q$ is calories, $c$ is specific heat, $m$ is mass, and $\Delta T$ is the temperature variation). The specific heat of the solution was assumed to be the same as water $\left(c_{\text {water }}=4.179 \mathrm{~J} / \mathrm{g}^{\circ} \mathrm{C}\right)$. The energy delivered to the solutions by NIR laser irradiation was determined as $J=W s$ (where $J$ is energy, $W$ is laser power, and $s$ is time). The photothermal transduction efficiency $\eta$ was then estimated as the ratio of the energy received to the energy delivered: $\eta=Q / J$. Figure 2(c) shows the photothermal transduction efficiency of GNR and GNP solutions as a function of mass concentration. Photothermal transduction efficiency increased with increasing GNR concentration (slope $=5.97$ ), whereas the efficiency was nearly constant for GNP solutions (slope $=0.08$ ). The sensitivity of efficiency to the GNR concentration suggests that GNR solutions are suitable as photothermal ablation agents.
Accordingly, the GNR solutions were then used as photothermal ablation agents for cancer cells. A schematic of the approach for photothermal ablation of cancer cells is shown in Figure 3(a). To evaluate GNRs as potential cancer cell photothermal ablation agents, hexadecyltrimethylammonium bromide (CTAB) on the surface of the GNRs must be modified because $\mathrm{CTAB}$ molecules exhibit a cytotoxic phenomenon and cellular function interference due to its strong electric potential [15]. ${ }^{1} \mathrm{H}-\mathrm{NMR}$ spectra were obtained to confirm the chemical structure of PGNRs (Figure 3(b)). The characteristic peaks of CTAB (3.1, 1.2, and $0.8 \mathrm{ppm}$, black line) were not detected, whereas that of PEG (3.6 ppm, red line) was observed in the PGNR spectra (blue line). The peaks at $4.8 \mathrm{ppm}$ were characteristic of dideuterium oxide $\left(D_{2} O\right)$ peaks from the solvent. These results indicated that the CTAB molecules were successfully exchanged with CM-PEG-SH molecules.

Figure 4(a) shows a dark field image of untreated MDAMB-231 cells as a negative control. The MDA-MB-231 cells appeared dark blue in the dark field image. In Figure 4(b), the scattered light from internalized PGNRs was observed as a dark yellow spot. Images of live-dead stained cells are shown in Figure 5 using fluorescence microscopic imaging. Regions of dead cells stained with EthBr (red region) broaden with increasing laser power density and irradiation time. No damage was observed in samples that were not NIR irradiated, in samples irradiated with $5 \mathrm{~W} / \mathrm{cm}^{2}$ for 10 minutes, or irradiated with $40 \mathrm{~W} / \mathrm{cm}^{2}$ for 30 minutes on MDA-MB-231 cells without PGNRs loading. In samples irradiated with $10 \mathrm{~W} / \mathrm{cm}^{2}$ for 10 minutes, $20 \mathrm{~W} / \mathrm{cm}^{2}$ for 5 minutes, or $20 \mathrm{~W} / \mathrm{cm}^{2}$ for 10 minutes, black dark spots in the calcein AM staining images (first row) and red spots in the EthBr staining images (second row) were observed. The size of the spots did not match the $2 \mathrm{~mm}$ spot size of the laser. The laser irradiation spots were bigger than the black dark spot and the red spot in the calcein AM and $\mathrm{EthBr}$ stained images, respectively, most likely due to the Gaussian nature of the irradiating beam, which causes cells at the periphery of the laser spot to receive less power [16]. Consequently, the minimum temperature for cell ablation was not achieved, so the black dark spots in the calcein AM staining images and the red spots in the EthBr staining images were smaller than laser irradiation spots. When the cells were irradiated at $40 \mathrm{~W} / \mathrm{cm}^{2}$ for 30 minutes, the black spot in the calcein AM images was larger than the laser irradiation spot, indicating that the heat generated by the PGNRs increased the temperature above that necessary for cell death, and cells in adjacent areas outside the laser spot diameter were also ablated as the excessive heat dissipated away from the PGNRs. In the merged images (the third row), the doughnut-shaped black areas suggest that PGNR-generated heat caused cell shrinkage [16].

We suggest that it is necessary to integrate and optimize a system for investigation about photothermal effect of photothermal agents, uptake efficiency of photothermal agents toward the specific cell lines, and cell viability assay after light irradiation (Figure 6). Thus, we assembled techniques to observe the above-mentioned phenomenon in one place. 


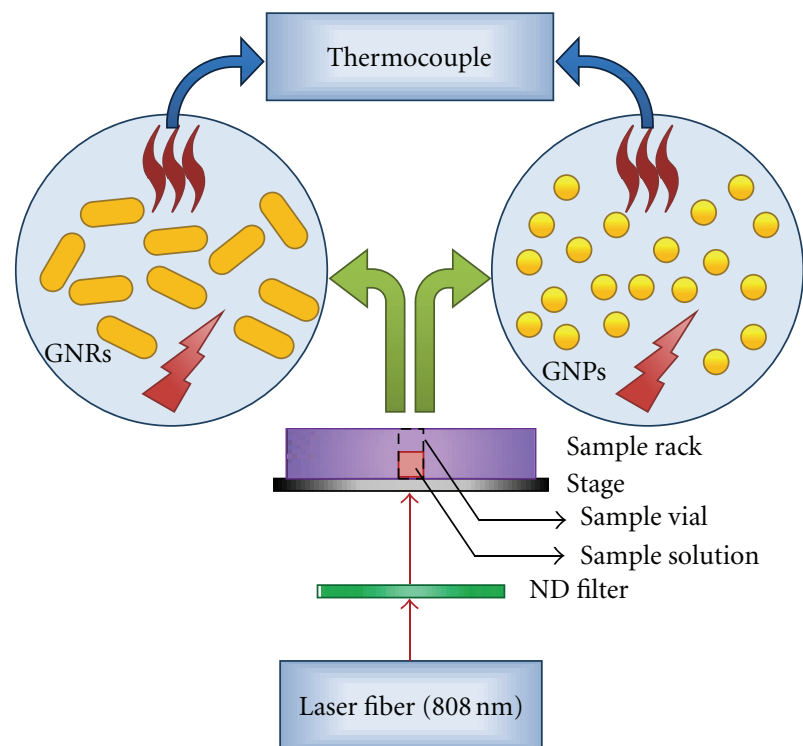

(a)
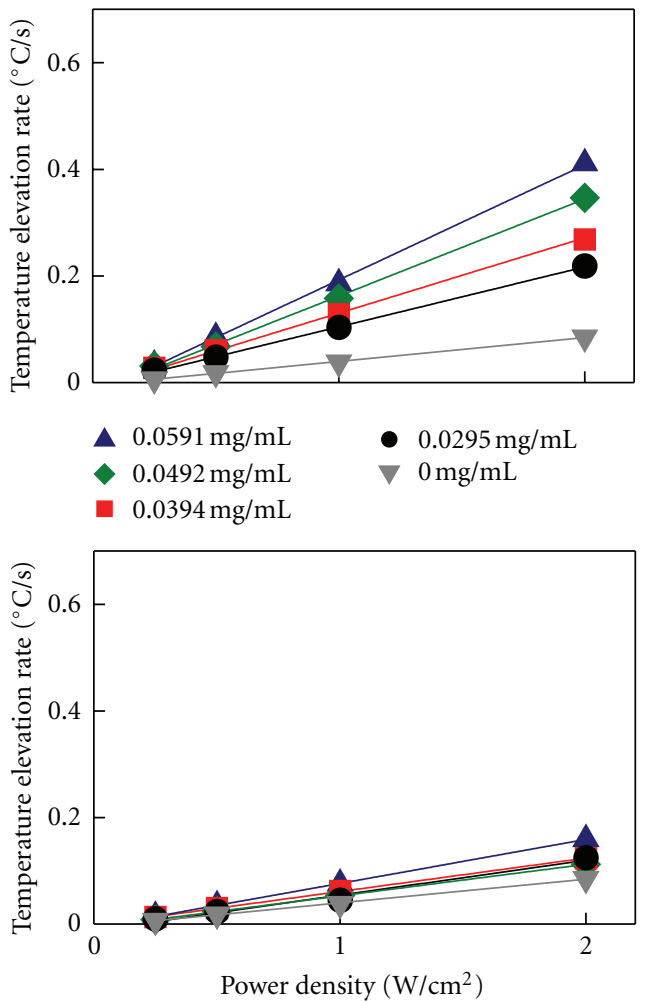

- $0.1 \mathrm{mg} / \mathrm{mL}$

$0.05 \mathrm{mg} / \mathrm{mL}$

$0.025 \mathrm{mg} / \mathrm{mL}$

(b)

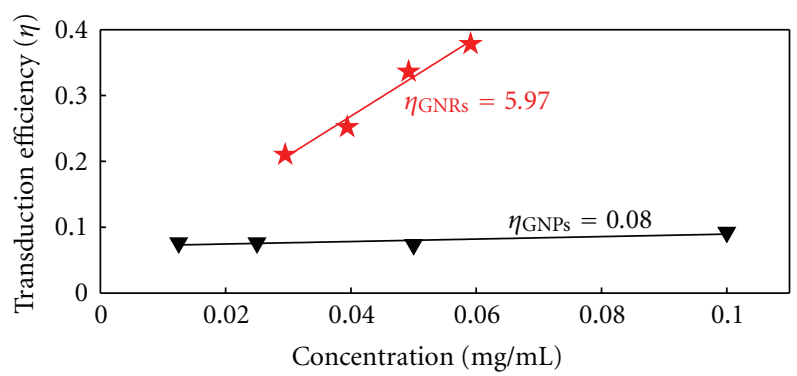

(c)

Figure 2: (a) Schematic illustration of temperature measurement system. (b) Temperature elevation rate as a function of power density for each GNR and GNP concentration; black: $0.0295 \mathrm{mg} / \mathrm{mL}$, red: $0.0394 \mathrm{mg} / \mathrm{mL}$, green: $0.0492 \mathrm{mg} / \mathrm{mL}$, and blue: $0.0591 \mathrm{mg} / \mathrm{mL}$ for GNRs; black: $0.0125 \mathrm{mg} / \mathrm{mL}$, red: $0.025 \mathrm{mg} / \mathrm{mL}$, green: $0.05 \mathrm{mg} / \mathrm{mL}$, and blue: $0.1 \mathrm{mg} / \mathrm{mL}$ for GNPs. (c) Photothermal transduction efficiencies of GNR and GNP solutions as a function of mass concentrations; black: GNPs, red: GNRs. Inserted numbers are slope values of each fitted graph.

Firstly, we designed optimized optical system for NIR laser irradiation. The wavelength of NIR laser was $808 \mathrm{~nm}$ and GNR have highest absorption in this wavelength, so this wavelength was suitable to investigate the photothermal effect of GNRs as photothermal agents. Second, we measured temperature of the samples in quasi-insulated system using thermocouple. The temperature elevation rate was analyzed depending on sample concentration and irradiated power density, and then, we calculated photothermal transduction efficiency using simple mathematical methods for each sample. Third, we conducted dark field imaging for confirmation of GNR uptake efficiency toward the cancer cells. The MDA-MB-231 cells appeared dark blue, whereas internalized PGNRs were observed as a dark yellow spot. The dark field image analysis was reliable method for assessment of uptake efficiency as nanoparticles and cells have different light scattering colors. Finally, we classified live and dead cells using fluorescence images that have distinct color 


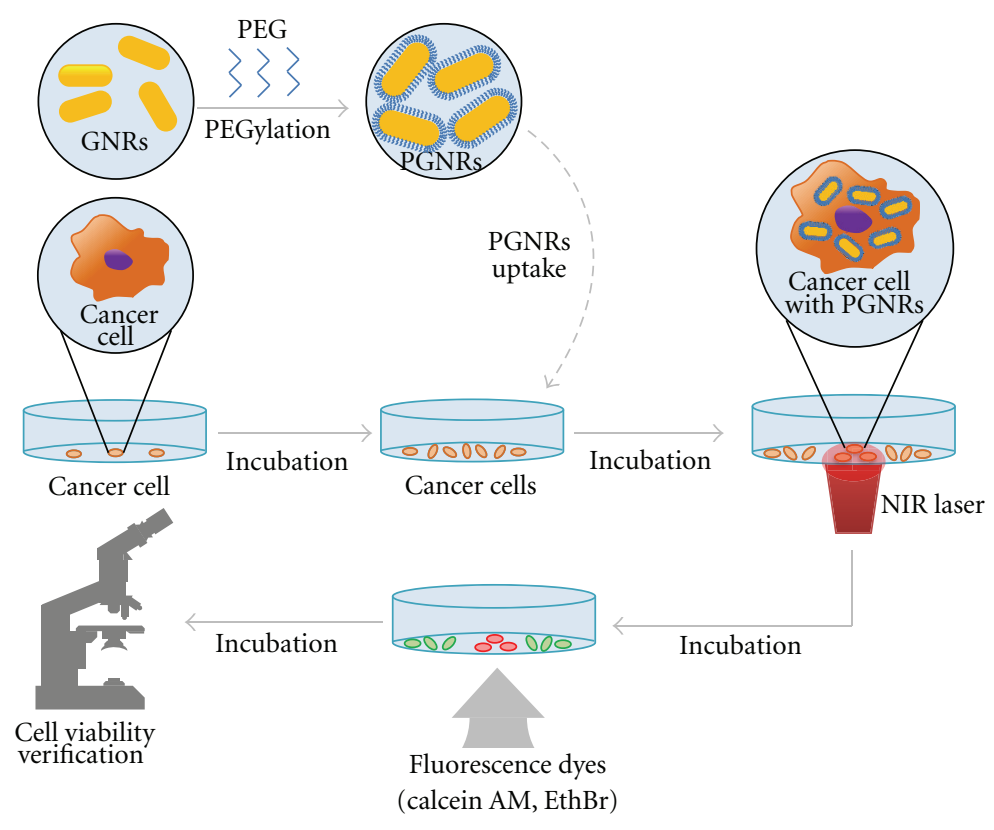

(a)

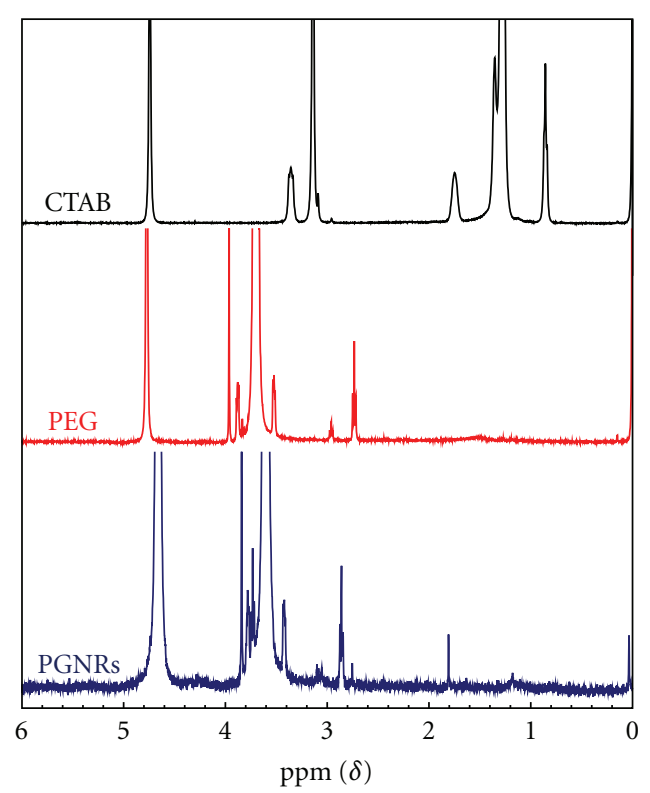

(b)

FIgURe 3: (a) Schematic of photothermal ablation with GNRs for cancer cells. (b) Proton-nuclear magnetic resonance $\left({ }^{1} \mathrm{H}-\mathrm{NMR}\right)$ spectrum; black: CTAB, red: PEG, and blue: PGNRs.

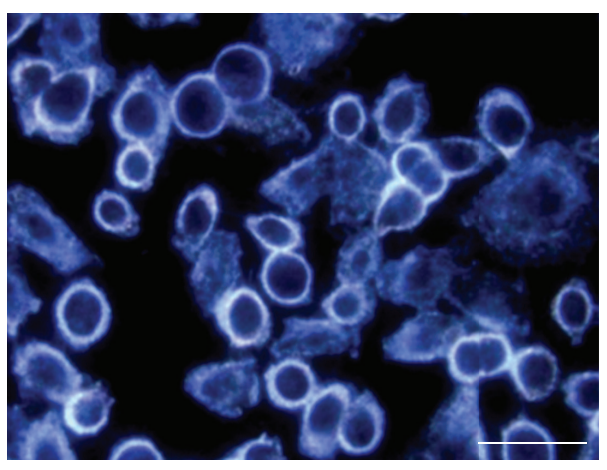

(a)

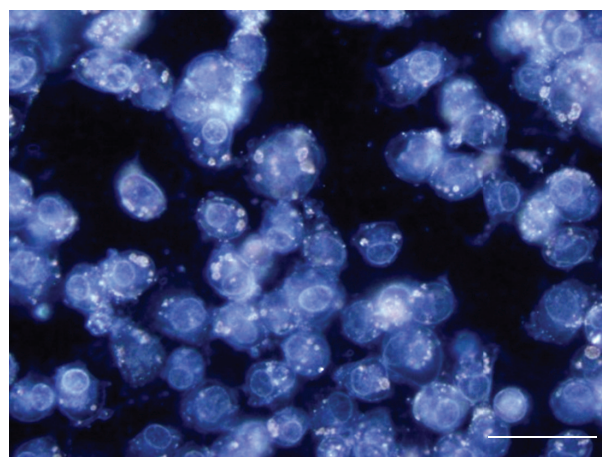

(b)

FIGURE 4: Dark field image of (a) nontreated MDA-MB-231 cells and (b) MDA-MB-231 cells treated with PGNRs. Scale bars: $20 \mu \mathrm{m}$. (c) Fluorescence images of MDA-MB-231 cells treated with PGNRs after NIR laser irradiation. Dotted lines mean NIR laser irradiated spot. Beam diameter of NIR laser is $2 \mathrm{~mm}$. All scale bars: $400 \mu \mathrm{m}$.

differences according to cell viability. Moreover, we show that modulation of the degree for cell viability according to GNRs concentration and irradiation laser power. We propose a series of technique processes for assessment of photothermal effect at once because these techniques are essential for execution and evaluation of photothermal therapy.

\section{Conclusions}

In conclusion, we modulated the photothermal transduction capabilities of GNRs and GNPs solutions for the ablation of cancer cells. The temperature elevations induced by different concentrations of GNRs and GNPs solutions subjected to different NIR laser power densities were measured. The temperature elevation rates of GNR and GNP solutions were calculated, and the efficiency of photothermal transduction was determined as a function of mass concentration. Finally, GNR-induced photothermal ablation was applied successfully to cancer cells at various NIR laser power densities and irradiation times and was characterized with live-dead cell staining. These results demonstrate the differences in photothermal transduction efficiency between GNRs and GNPs solutions and suggest that photothermal ablation of cancer cells is sensitive to this efficiency as well as the irradiation conditions. Based on these results, we propound a system for photothermal effect analysis. A set of separate techniques 


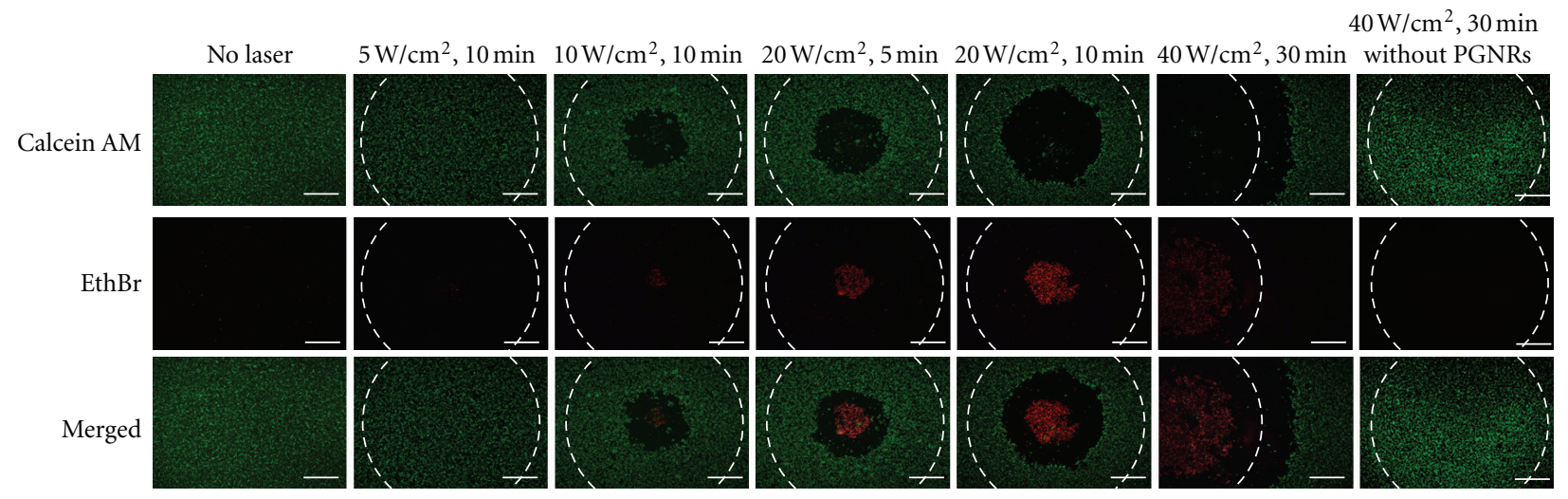

FIGURE 5: Fluorescence images of MDA-MB-231 cells treated with PGNRs after NIR laser irradiation. Dotted lines mean NIR laser irradiated spot. Beam diameter of NIR laser is $2 \mathrm{~mm}$. All scale bars: $400 \mu \mathrm{m}$.

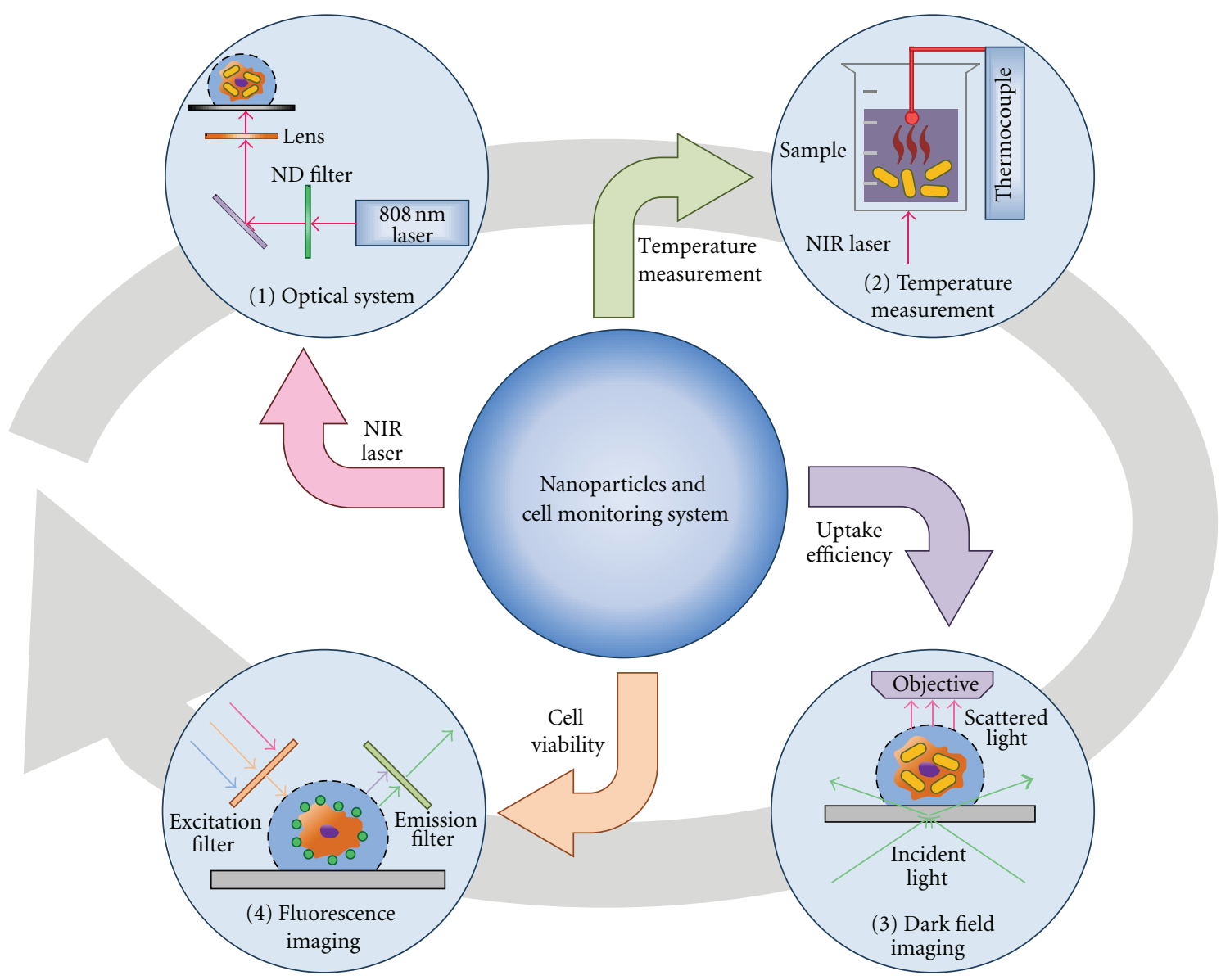

FIGURE 6: Schematic illustration of developed system for investigation of photothermal effect.

converged to a point with the aim of accurate evaluation for photothermal therapy. This system will present a way about precise photothermal therapy evaluation.

\section{Conflict of Interests}

The authors do not have a direct financial relation with the commercial identity mentioned in our paper (Shimadzu,
JEOL Ltd., Thermo Electron Corporation, JENOPTIK, Fluke, and Olympus).

\section{Acknowledgments}

This work was supported by the National Research Foundation (NRF) Grant funded by Korea Government, Ministry of Education and Science Technology (MEST) (2011-0026073). 
This study was supported by a grant from the Korean Health Technology R\&D Project of the Ministry for Health, Welfare \& Family Affairs, Republic of Korea (A101954).

\section{References}

[1] J. Yang, J. Lee, J. Kang et al., "Smart drug-loaded polymer gold nanoshells for systemic and localized therapy of human epithelial cancer," Advanced Materials, vol. 21, no. 43, pp. 4339-4342, 2009.

[2] R. Weissleder, "A clearer vision for in vivo imaging," Nature Biotechnology, vol. 19, no. 4, pp. 316-317, 2001.

[3] X. Huang, P. K. Jain, I. H. El-Sayed, and M. A. El-Sayed, "Gold nanoparticles: interesting optical properties and recent applications in cancer diagnostics and therapy," Nanomedicine, vol. 2, no. 5, pp. 681-693, 2007.

[4] H. Park, J. Yang, J. Lee, S. Haam, I. H. Choi, and K. H. Yoo, "Multifunctional nanoparticles for combined doxorubicin and photothermal treatments," ACS Nano, vol. 3, no. 10, pp. 2919-2926, 2009.

[5] P. K. Jain, I. H. El-Sayed, and M. A. El-Sayed, "Au nanoparticles target cancer," Nano Today, vol. 2, no. 1, pp. 18-29, 2007.

[6] P. Chakravarty, R. Marches, N. S. Zimmerman et al., "Thermal ablation of tumor cells with antibody-functionalized single-walled carbon nanotubes," Proceedings of the National Academy of Sciences of the United States of America, vol. 105, no. 25, pp. 8697-8702, 2008.

[7] X. Huang, P. K. Jain, I. H. El-Sayed, and M. A. ElSayed, "Plasmonic photothermal therapy (PPTT) using gold nanoparticles," Lasers in Medical Science, vol. 23, no. 3, pp. 217-228, 2008.

[8] H.-C. Huang, K. Rege, and J. J. Heys, "Spatiotemporal temperature distribution and cancer cell death in response to extracellular hyperthermia induced by gold nanorods," ACS Nano, vol. 4, no. 5, pp. 2892-2900, 2010.

[9] S. Link and M. A. El-Sayed, "Shape and size dependence of radiative, non-radiative and photothermal properties of gold nanocrystals," International Reviews in Physical Chemistry, vol. 19, no. 3, pp. 409-453, 2000.

[10] R. Choi, J. Yang, J. Choi et al., "Thiolated dextran-coated gold nanorods for photothermal ablation of inflammatory macrophages," Langmuir, vol. 26, no. 22, pp. 17520-17527, 2010.

[11] J. Yang, K. Eom, E. K. Lim et al., "In situ detection of live cancer cells by using bioprobes based on Au nanoparticles," Langmuir, vol. 24, no. 21, pp. 12112-12115, 2008.

[12] J. Choi, J. Yang, D. Bang et al., "Targetable gold nanorods for epithelial cancer therapy guided by near-IR absorption imaging," Small, vol. 8, no. 5, pp. 746-753, 2012.

[13] S. Link, M. B. Mohamed, and M. A. El-Sayed, "Simulation of the optical absorption spectra of gold nanorods as a function of their aspect ratio and the effect of the medium dielectric constant," The Journal of Physical Chemistry B, vol. 103, no. 16, pp. 3073-3077, 1999.

[14] F.-Y. Cheng, C.-T. Chen, and C.-S. Yeh, "Comparative efficiencies of photothermal destruction of malignant cells using antibody-coated silica@Au nanoshells, hollow Au/Ag nanospheres and Au nanorods," Nanotechnology, vol. 20, no. 42, Article ID 425104, 2009.

[15] A. M. Alkilany, P. K. Nagaria, C. R. Hexel, T. J. Shaw, C. J. Murphy, and M. D. Wyatt, "Cellular uptake and cytotoxicity of gold nanorods: molecular origin of cytotoxicity and surface effects," Small, vol. 5, no. 6, pp. 701-708, 2009.
[16] L. B. Carpin, L. R. Bickford, G. Agollah et al., "Immunoconjugated gold nanoshell-mediated photothermal ablation of trastuzumab-resistant breast cancer cells," Breast Cancer Research and Treatment, vol. 125, no. 1, pp. 27-34, 2011. 

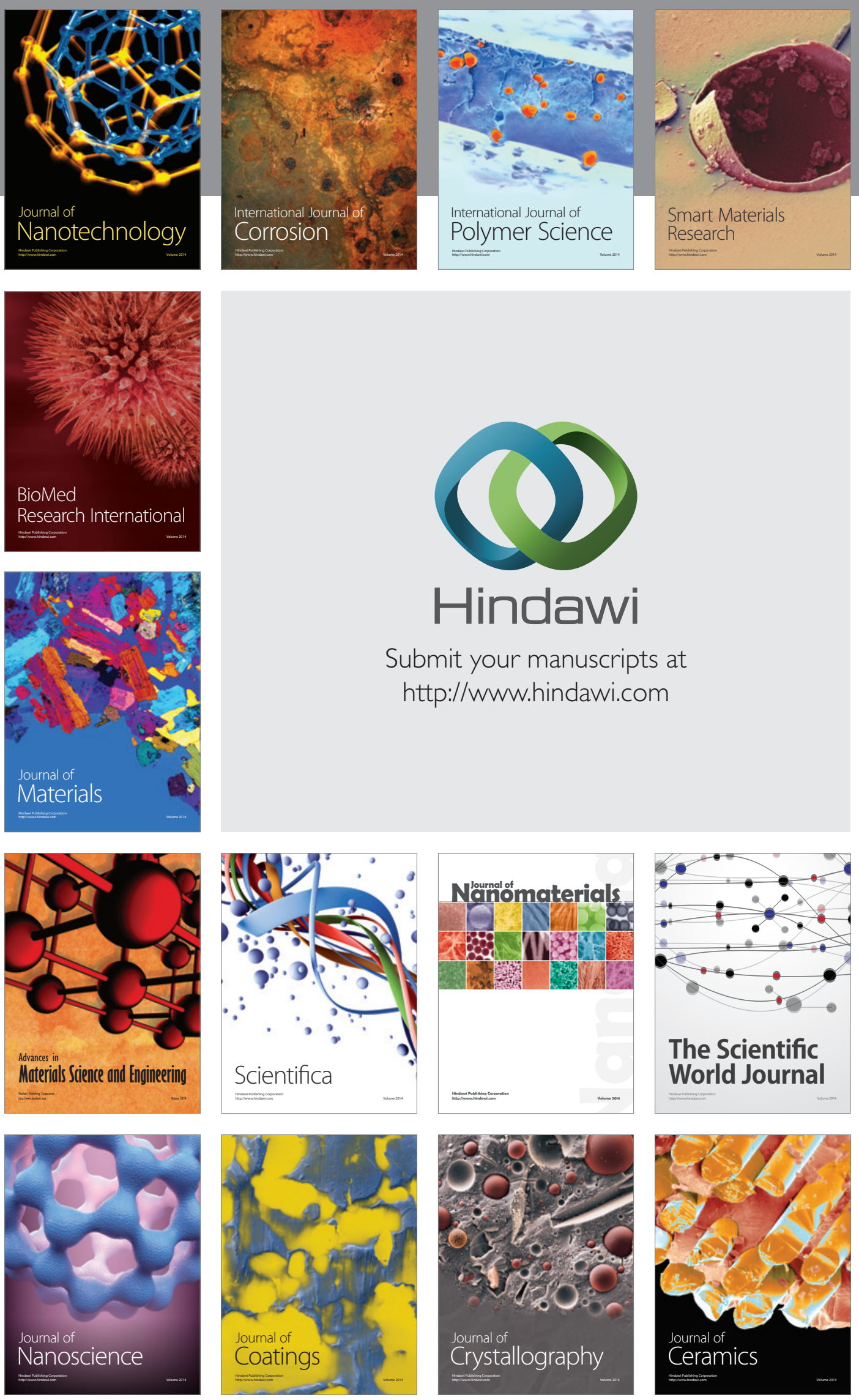

The Scientific World Journal

Submit your manuscripts at

http://www.hindawi.com

\section{World Journal}

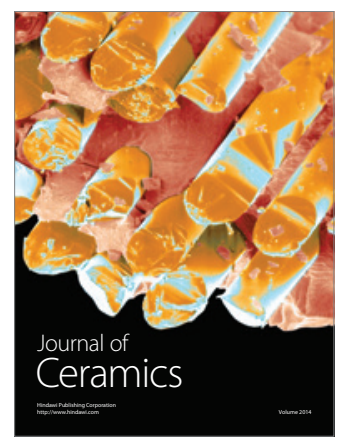

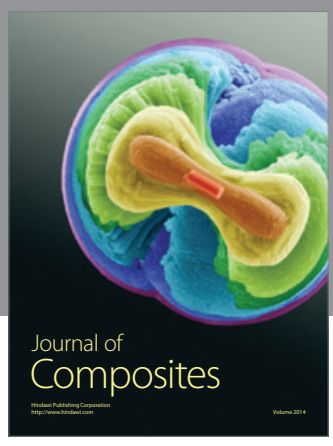
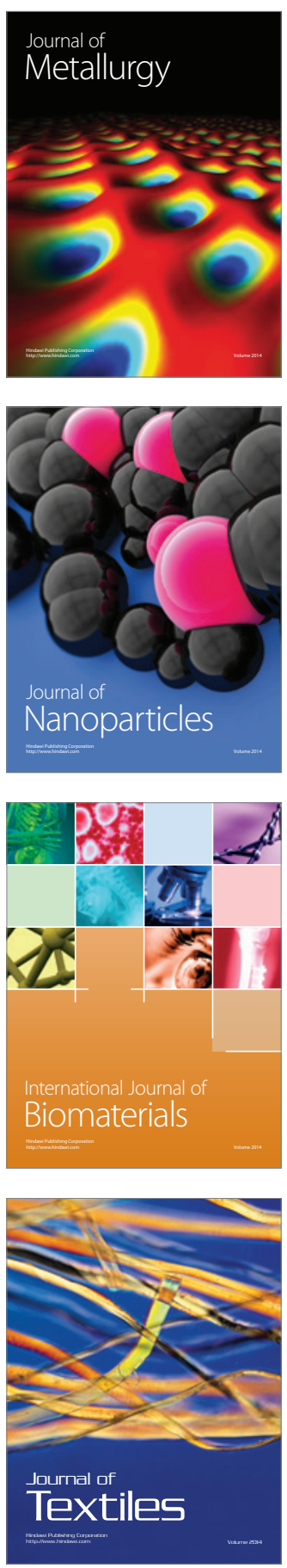